\title{
An Association Between Implementing Trauma-Informed Care and Staff Satisfaction
}

\author{
Travis W. Hales \\ Thomas H. Nochajski \\ Susan A. Green \\ Howard K. Hitzel \\ Elizabeth Woike-Ganga
}

\begin{abstract}
Despite its widespread adoption there is limited research on the influence of trauma-informed care (TIC). The current study examined the impact of implementing TIC on the satisfaction of agency staff by comparing the results of a satisfaction survey taken in January of 2014, a month prior to the agency's implementation of TIC, and again twelve months later. As collaboration, empowerment, and self-care are primary components of a TIC organizational approach, its implementation was expected to increase staff satisfaction. Following the implementation of TIC, agency staff reported higher scores on all but one of the six satisfaction survey factors. Increases in staff satisfaction have been associated with better staff retention rates, increased organizational commitment and better performance. In consequence, TIC implementation is associated with increased staff satisfaction, and may positively influence organizational characteristics of significance to social service agencies.
\end{abstract}

Keywords: Trauma-informed care; evidence; implementation; staff satisfaction

In recent years, trauma-informed care (TIC) has become increasingly adopted across disciplines and communities of practice (Hodgdon, Kinniburgh, Gabowitz, Blaustein, \& Spinazzola, 2013; Muskett, 2014). The rising interest in TIC is a natural consequence of repeated investigations demonstrating the prevalence of trauma in society (Kilpatrick et al., 2013), and the negative impact exposure to traumatic events has on a host of social, physical and mental health outcomes (Kuo, Goldin, Werner, Heimberg, \& Gross, 2011; Park et al., 2014). TIC has three primary components: the realization of the pervasiveness of trauma; recognition of how trauma influences all persons involved in the receipt, delivery, and administration of programs; and being responsive to trauma survivors needs by implementing this knowledge into the organization's policies, procedures, practices and culture (Substance Abuse and Mental Health Services Administration, 2014). Despite the increase in implementation efforts the effectiveness of TIC remains largely unexplored, particularly in regards to its impact on the adopting system and accompanying staff members. The current study begins to overcome this gap by examining the effects of implementing TIC on the satisfaction of agency staff.

The current study is situated within two primary streams of research: research on the effects of implementing TIC and research on staff satisfaction. In regards to research on

\footnotetext{
Travis W. Hales, MSW, is PhD Candidate, School of Social Work, University at Buffalo, State University of New York, Buffalo, NY 14214-8004. Thomas H. Nochajski, PhD, is Research Professor, School of Social Work, University at Buffalo. Susan A. Green, LCSW, is Clinical Associate Professor, School of Social Work, University at Buffalo. Howard K. Hitzel, PsyD, MPA is President, BestSelf Behavioral Health, Buffalo, NY 14202. Elizabeth Woike-Ganga, LCSW-R, is Chief Operating Officer, BestSelf Behavioral Health.
} 
the impact of TIC, it is important to differentiate this research from that conducted on trauma in general and on trauma-specific treatments. There has been extensive research conducted on the pervasiveness of trauma and its influence on personal development, perhaps most notably of which is the Adverse Childhood Experiences (ACE) Study (Felitti et al., 1998). The ACE study included 17,337 men and women and found that $63.9 \%$ of the sample experienced at least one adverse childhood experience (Center for Disease Control and Prevention, n.d.). The primary finding in the study was the association between the number of ACEs participants experienced with risk of alcoholism and substance use, heart disease, sexually transmitted diseases, suicide attempts, adolescent pregnancy, and risk for sexual violence among others (Felitti et al., 1998). Further, there has been research on specific interventions that treat a variety of trauma and comorbid trauma and substance use or mental health disorders, including Trauma Focused Cognitive Behavioral Therapy (TFCBT; Seidler \& Wagner, 2006), Seeking Safety (Najavits, Weiss, Shaw, \& Muenz, 1998), and Cognitive Processing Therapy (CPT; Resick, Nishith, Weaver, Astin, \& Feuer, 2002). However, there has been extremely limited research on the effects of trauma-informed organizational approaches (Kusmaul, Wilson, \& Nochajski, 2015).

To differentiate TIC from trauma and trauma-specific treatment, trauma-informed approaches such as Harris and Fallot's (2001; Fallot \& Harris, 2008) five-dimensional model and Sandra Bloom's (2013) Sanctuary model are defined by the alteration of systems to ensure their physical and social environments are supportive and conducive to the recovering person that has a history of trauma. This includes creating atmospheres where all persons feel safe and relationships are based on shared assumptions, collaboration and trustworthiness (Madsen, Blitz, McCorkle, \& Panzer, 2003). Trauma-informed systems recognize the pervasiveness of trauma and revamp formal and informal organizational properties (such as structure, culture, climate, policies, procedures and practices) to ensure trauma-sensitivity and promote growth and empowerment (Bloom, 2013; Harris \& Fallot, 2001). Note that people as opposed to clients or service-users are referred to throughout this discussion because, contrary to research emphases in the literature, trauma-informed approaches are designed to have a positive influence on the staff of service organizations as well as clients. Nevertheless, the limited evidence that exists on implementing TIC approaches has focused primarily in its influence on clients (e.g., Morrissey et al., 2005).

\section{Research on the Impact of TIC}

The published evidence on the effects of TIC on clients has been promising. There are two bodies of research in this area: research exploring the effects of TIC reducing the number of seclusions and restraints in psychiatric inpatient settings and research exploring the effects of TIC on substance use and mental health outcomes. Research on seclusion and restraint reduction began with the work of Janice LeBel and colleagues from Massachusetts' Department of Mental Health who demonstrated that seclusion and restraints could be greatly reduced through systematic change efforts (LeBel \& Goldstein, 2005; LeBel et al., 2004). Further, LeBel and Goldstein (2005) discovered that the reduction in seclusion and restraints led to decreases in employee sick time, turnover, workers' compensation, injuries to both staff and clients, and recidivism in clients. However, neither published study explicitly refers to the intervention as being part of a 
trauma-informed approach. While seclusion and restraints are the prototypical examples of re-traumatization in service systems, because TIC is not explicitly referred to in LeBel's work it is unclear whether or not the reduction and subsequent effects are attributable to part of a broader TIC initiative. To address this gap in research, Azeem, Aujla, Rammerth, Binsfeld and Jones (2011) assessed the influence of a TIC model developed by the National Association of State Mental Health Program Directors (NASMHPD). Through the implementation of various components of TIC including physical and social (i.e., cultural) changes to the environment, staff training on trauma (i.e., prevalence and effects) and trauma-informed care, prioritizing collaboration between staff and residents, and debriefing with staff and clients after a crisis, the TIC approach led to a downward trend in seclusions and restraints (Azeem et al., 2011).

In regards to the second stream of research investigating the effects of implementing TIC on substance use and psychiatric outcomes, the Women, Co-occurring Disorders, and Violence Study (WCDVS) is the singular example (described in Morrissey et al., 2005). The WCDVS assessed the impact of core trauma-informed services (including collaboration between service providers and recipients in treatment planning and goal development, resource coordination, staff training on trauma, and crisis intervention) and integrated substance use, trauma and mental health counseling on substance use, mental health, and trauma symptom outcomes (Morrisey et al., 2005). The intervention was found to have positive and significant effects on substance use and mental health outcomes and a significant reduction in trauma symptoms. However, the significant effects were primarily attributable to the integrated treatment as opposed to the core TI services. Future research is required to determine the underlying mechanism of change in the study and to see if the findings are replicated. (Morrisey et al., 2005)

The only other studies in the literature examining TIC's effects are Kramer, Sigel, Connors-Burrow, Savary and Tempel (2013) and Kusmaul and colleagues (2015). Kramer et al. investigated the impact of a two-day training based on the National Child and Traumatic Stress Network's training for child welfare. The outcomes of the Kramer et al. study were limited to knowledge on TIC and supervisory behaviors (measured through self-report on survey items) which were assessed at baseline, immediately following the final training, and a three-month follow-up. The post-test and three-month follow-up displayed significant increases in TIC knowledge and supervisory behaviors supporting its implementation (Kramer et al., 2013). Kusmaul et al. (2015) explored differences in staff perceptions of the service environment following the implementation of TIC based on the principles of Harris and Fallot (2001). Kusmaul et al. discovered that staff members holding Master's level degrees had more positive experiences of the service environment following TIC implementation than staff members holding a Bachelor's degree. Further, perceptions of the service environment also differed as a function of position, with administrators having the most positive perceptions of the service environment, followed by line staff, and then support staff. Both of these findings were consistent across Harris and Fallot's (2001) five domains. While further research is needed to explain these differences, what is evident is that persons higher in the organizational hierarchy and persons holding a Master's level degree have more positive experiences of the service environment than their counterparts. This is the extent of published knowledge on the 
effects of TIC on both staff and client outcomes. While the evidence is severely limited, especially in regards to the impact of TIC approaches on staff, initial reports are promising. There is however a need for continued research on the effects of TIC approaches.

\section{Research on Staff Satisfaction and its Relationship with Trauma-Informed Care}

On the surface, the relationship between TIC and staff satisfaction may be predicated on the emphasis trauma-informed approaches place on staff self-care. That is, a core component of all prevailing TIC models is the creation of safe and trusting atmospheres to avoid the re-traumatization of both staff and consumers of service (Bloom, 2013; Fallot \& Harris, 2008). As the organization becomes more supportive of direct-care and support staff, it is likely the organization will experience a positive increase in the overall satisfaction of its agents (Babin \& Boles, 1996; Griffin, Patterson, \& West, 2001).

Above and beyond self-care, the core components of trauma-informed approaches are also expected to have a significant positive influence on staff's satisfaction with the organization. The elements of safety, trustworthiness, collaboration, choice and empowerment from both Fallot and Harris (2008) and Bloom's (2013) Sanctuary model are expected to enhance staff's experiences of satisfaction with the agency. Through collaboration, where all staff members are treated with equal regard and importance, the problems originating from hierarchy, power and authority may be overcome. Collaboration, freedom and autonomy of staff throughout the organization are expected to increase staff member's sense of commitment and responsibility for the organization's welfare. For empirical support of these hypotheses, both Babin and Boles (1996) and Strand and Dore (2009) discovered that the perceptions of supervisory support were positively associated with increases in staff satisfaction. Further, Brown and Peterson (1993) demonstrated how staff satisfaction predicted organizational commitment, which in turn has been associated with higher staff retention rates (Meyer, Stanley, Herscovitch, \& Topolnytsky, 2002; Strand, Spath, \& Bosco-Ruggiero, 2010), and staff satisfaction and organizational commitment have both been tied to organizational performance (Koys, 2001; Meyer et al., 2002).

\section{The Current Study}

The current study assessed the influence of a TIC implementation approach on staff satisfaction by comparing the results of a satisfaction survey from two time points. The first assessment was taken in January of 2014, a month prior to the organization's implementation of TIC, while the second assessment was conducted twelve months following the organization's implementation (January of 2015). The satisfaction surveys were conducted by an external agency specializing in workforce development. All staff members within the organization were administered the online survey. Following the completion of both surveys, a brief report was provided to the agency summarizing the results and comparing the 2014 and 2015 surveys. Because self-care and the creation of supportive cultures are central to the implementation of TIC, we expected there to be an increase in staff satisfaction following the twelve-month implementation process. 


\section{Methods}

\section{Implementation Model}

The implementation model consisted of a series of phases. The first phase included an initial baseline assessment of the organization on trauma-informed care. Two assessment tools were utilized: an instrument that assessed the culture and climate (described in Hales, Kusmaul, \& Nochajski, 2016) of the organization in the context of Harris and Fallot's (2001) five-principles, and an instrument that assessed the organization's policies, practices and procedures (described in Gaurino, Soares, Konnath, Clervil, \& Bassuk, 2009). Following the assessment, all staff members within the organization were trained on the basic tenets of trauma and trauma-informed care. The TIC trainings were based on Harris and Fallot's five-dimensional model. Professional staff holding advanced degrees delivered the trainings using a protocol developed by the authors.

After the initial training, the assessment data were used to inform each of the programs of their relative strengths and weaknesses in regard to formal (policies, practices and procedures) and informal (culture and climate) organizational elements. This information was incorporated into all follow-up training sessions, which were tailored to the particular program's needs and strengths. Follow-up sessions recurred on a monthly basis following the initial training. Supervisors were mentored and coached on how to incorporate the implementation of TIC into weekly supervision. To enhance sustainability, staff members across the organizational hierarchy were trained on how to conduct the initial training and follow-up sessions, which enabled the organization to deliver information to new employees, provide refresher courses to programs seeking them, and continue monthly consultations on the implementation of TIC without the aid of the consultants.

In addition to the trainings, a sub-group of clinical staff were trained on the delivery of Seeking Safety, an evidence-based treatment for persons experiencing comorbid substance-use and post-traumatic stress disorders (described in Najavits, 2002). This subgroup of personnel received formal training on the intervention's delivery, treatment protocols to ensure fidelity, and monthly consultations to discuss the implementation processes. Thus, the implementation model included the assimilation of a trauma-specific treatment in addition to training and overseeing the implementation of a trauma-informed organizational approach.

\section{Instrument Description}

The Business Insight survey from WorkplaceDynamics (n.d.) consisted of a 5-point Likert scale ranging from 1 (strongly disagree) to 5 (strongly agree). While the survey has been administered to over 40,000 organizations, the authors do not list the scale's psychometric properties (WorkplaceDynamics, n.d.). The survey measured staff satisfaction on two primary domains and six sub-domains. The two primary domains consisted of organizational health, which included direction of the agency, execution of the agency's policies and procedures, and connection with the workplace, and staff's work positions, which included perceptions of their work, relationship with their manager, and their pay/benefits. To describe the subdomains, direction of the agency inquired into staff 
perceptions of the overall direction the agency, the competencies of leadership, and organizational values. Execution of the agency's policies and procedures measured satisfaction with organizational efficiency, innovation, and being informed of administrative decisions. Connection with the workplace assessed whether staff believed their role in the agency was valued by others in addition to whether or not they participated in meaningful work. The connection with the work factor inquired into staff experiences with flexibility in their work roles, receipt of appropriate training to successfully execute role functions, and whether or not their position had met their expectations. The management factor assessed staffs' relationships with their managers, including whether their manager was sensitive to their needs and concerns, fostered their growth, and enhanced their performance. Lastly, pay/benefits inquired into how fair staff believed their wages and benefit packages were. Each of the six domains ranged from two to four items, with a total of 19 items in the scale. Individual items that experienced the most improvement across time points were included in the report. The individual items were: "new ideas are encouraged at the agency" (encourage new ideas); "senior managers understand what is really happening at the agency" (mgmt. understanding); and "my manager cares about my concerns" (mgmt. care and concern).

\section{Sample}

The sample included staff from a private, not-for-profit 501(c)3 corporation that provides a range of services for individuals with mental health and/or substance use disorders. These services include: outpatient programs for adolescents and adults with substance use, mental health, and co-occurring disorders; personalized recovery oriented services (PROS); assertive community treatment (ACT) teams; residential programs for pregnant and parenting women with substance use disorders and chronically homeless persons with mental health diagnoses; homeless outreach services; mobile mental health teams; permanent supportive housing services; and vocational and supported employment services.

During the survey period, the number of staff members across all programs ranged from 212 to 238. For 2014, a total of 147 out of 212 employees completed the survey (69 percent completion rate). For 2015, 168 out of 238 employees completed the instrument (71 percent completion rate). The survey was administered to all staff across the organization's vertical hierarchy and throughout the various programs. Specific demographic information could not be reported due to their lack of inclusion in the satisfaction survey's report.

\section{Analyses}

Due to the data being collected by an external agency, raw data could not be obtained. The report only contained the aggregate means for the agency on the six factors of satisfaction across the two time points. It was not possible to match participants across time periods, and because the standard deviations were not provided, standard errors and significance tests could not be computed. In consequence, the analyses for comparing the 2014 and 2015 data were limited to assessing mean differences on each of the satisfaction's subscales. The differences between the years were assessed by comparing Likert-point and 
percentile differences across time points. Further, the comparisons were primarily limited to the six subscales, as only those individual items that experienced dramatic increases or decreases were provided in the report.

\section{Results}

Following the implementation of TIC, the average scores on five of the six factors of staff satisfaction increased. Presented in Table 1 below, the largest increases were in execution of the organization's objectives, followed by staffs' relationships with their manager, staffs' connection with the workplace, then staff's perceptions of the work. Direction of the agency increased but was more modest relative to the other domains. The only scale which did not increase was pay/benefits, although it also experienced the smallest absolute difference/percentage change.

Table 1. Comparison of Average Satisfaction Subscale Scores for 2014 and $2015(\mathrm{n}=168)$

\begin{tabular}{|c|c|c|c|c|}
\hline Scales & 2014 (Mean) & 2015 (Mean) & $\begin{array}{c}\text { Absolute } \\
\text { Difference }\end{array}$ & $\begin{array}{c}\text { Percentage } \\
\text { Change* }\end{array}$ \\
\hline Execution & 3.83 & 4.14 & +0.31 & $+8.09 \%$ \\
\hline Connection & 4.35 & 4.57 & +0.22 & $+5.06 \%$ \\
\hline Direction & 4.62 & 4.76 & +0.14 & $+3.03 \%$ \\
\hline Manager & 4.55 & 4.81 & +0.26 & $+5.71 \%$ \\
\hline Work & 4.21 & 4.39 & +0.18 & $+4.27 \%$ \\
\hline Pay/Benefits & 3.51 & 3.44 & -0.07 & $-1.99 \%$ \\
\hline
\end{tabular}

In regard to the individual item examination, a total of three indicators were reported, three of which were directly relevant to the implementation of TIC. To begin, the encouragement of new ideas by administration increased from an average of 4.10 to 4.52 (.42 Likert-points; +10.24 percent). The next item refers to staffs' perceptions of senior managers understanding the reality of daily programmatic affairs. This item also increased by approximately ten percent (from 3.64 to 4.01 Likert points). Lastly, staff reported an increase in the extent that management cares about their concerns (from 4.63 to 4.94 Likert points; + 6.70\%).

Table 2. Comparison of Average Item Scores for 2014 and $2015(\mathrm{n}=168)$

\begin{tabular}{lcccc}
\hline Items & 2014 (Mean) & 2015 (Mean) & $\begin{array}{c}\text { Absolute } \\
\text { Difference }\end{array}$ & $\begin{array}{c}\text { Percentage } \\
\text { Change* }\end{array}$ \\
\hline Encourage new ideas & 4.10 & 4.52 & +0.42 & $+10.24 \%$ \\
Mgmt. understanding & 3.64 & 4.01 & +0.37 & $+10.16 \%$ \\
Mgmt. care and concern & 4.63 & 4.94 & +0.31 & $+6.70 \%$ \\
\hline
\end{tabular}

\section{Discussion}

The current study found increases in staff satisfaction in five of the six satisfaction factors following the implementation of TIC. The most notable differences were in staff's satisfaction with their ability to execute the agency's executives, their relationship with 
management, and their connection to the workplace. These findings are theoretically plausible considering that trauma-informed approaches are expected to enhance collaboration across vertical hierarchies in addition to empowering staff throughout the organization by maximizing their experiences of control and autonomy (Bloom, 2013; Fallot \& Harris, 2008). By engaging members throughout the organization in collaborative approaches in the obtainment of organizational objectives, staff's experiences of commitment to the agency were expected to increase. The only factor which did not increase across time points was pay/benefits. This is plausible, considering that the second assessment of satisfaction was only conducted twelve months following implementation. While implementing TIC may be expected to save the organization material resources by retaining a greater percentage of the workforce and by providing more responsive services to clients, these changes may take a greater length of time to emerge. A body of research indicates that changes to organizational culture generally occurs over a period of time (Cameron \& Quinn, 2005), and the effects of implementing TIC on staff retention is expected to be a longer term outcome (Esaki et al., 2013).

Staff also reported increases in satisfaction with management's encouragement of new ideas and innovation. Administrative encouragement of new and better ways of performing their tasks is a critical element of empowerment, one of the primary principles of TIC. Staff also felt increases in management's ability to be understanding of the reality of day-to-day affairs. The principle of collaboration in TIC approaches requires that organizational hierarchies be flattened, minimizing the physical and social distance between front-line and senior staff members. Creating a more collaborative environment could enhance the communication and joint work efforts of management and direct-care staff. Staff also experienced an increase in satisfaction with management's concern over their general welfare, which is plausible considering trauma-informed approaches prioritize the experiences of direct-care staff by ensuring their physical and emotional safety.

As there is limited research on the influence of implementing trauma-informed approaches on staff, the positive association between the implementation of TIC and increases in staff satisfaction is an important finding. While the implementation of TIC only produced modest variations in staff satisfaction, it is important to note that the introduction of new technologies are frequently met with varied responses by staff members (e.g., Korunka, Scharitzer, Carayon, \& Sainfort, 2003). As a core component of trauma-informed organizations is an increase in staff control, autonomy, and collaboration, it is possible that staff experience an increase in responsibility over obtaining the organization's objectives. This increase in responsibility and power may have a negative influence on some staff member's satisfaction, particularly if staff prefer a more centralized organization where job functions and roles are more instructive and formalized. Further, while previous research has found moderate associations between staff autonomy and supervisory support with satisfaction (Griffin et al., 2001), to the best of our knowledge no studies that have assessed longitudinal differences in satisfaction scores provide neither mean difference scores or effect sizes to compare to the current study's findings.

Nevertheless, because all but one of the satisfaction scores increased, the current study's findings suggest the assimilation of trauma-informed care within organizational settings enhances staff satisfaction. Due to the limited evidence supporting the widespread 
adoption of trauma-informed approaches, this study provides preliminary support on the positive influence of TIC on agency staff. In addition, previous research has demonstrated that satisfaction with the workplace is a powerful antecedent of organizational commitment (Brown \& Peterson, 1993), and that organizational commitment is positively associated with employee retention (Meyer et al., 2002; Somers, 1995). As staff satisfaction and organizational commitment have been associated with enhanced performance (Koys, 2001; Meyer et al., 2002), TIC may also have positive influence on performance. These arguments suggest that the implementation of TIC, through increasing staff satisfaction, may positively influence organizational characteristics of significance to social service agencies.

\section{Limitations}

There were several limitations in the current study. A primary limitation is the absence of raw data, and the subsequent ability to match pre and post test responses. The absence of raw data limited the analyses to absolute mean and percentile differences. However, due to the limited knowledge base on the influence of TIC implementation, the association between TIC and staff satisfaction is an important discovery. Further, while association and temporal precedence were obtained, confounding factors that may have explained the differences in satisfaction surveys were not controlled for. For instance, Seeking Safety was implemented to a subset of staff during the same time that staff were implementing TIC throughout the agency. In consequence, it is unclear what elements of the intervention (i.e.,Seeking Safety, training, or consultation) influenced the aggregate differences in staff satisfaction. The relationship between TIC implementation and staff satisfaction is limited to one of association as opposed to causation. Further, because the second time point was only assessed twelve months following the initial implementation, it is possible that the effects of TIC on staff satisfaction were the result of increases in supervisory and organizational support, and increases in perceived competence as a result of the training and consultation sessions. The more subtle, informal changes to organizational culture and climate generally occur over a more extended period of time (Cameron \& Quinn, 2005; Esaki et al., 2013). The contributions of increasing staff's perceptions of safety, trust, choice, collaboration and empowerment on satisfaction would likely be evident had the follow-up assessment occurred at a later point in time. Lastly, a limitation of the current study is the absence of sample demographics.

\section{Future Research Directions}

Future research will explore the relationship between the implementation of TIC and additional staff and client characteristics. Additional research that involves tracking individual scores in satisfaction over time and controlling for the various intervention components will enable a test of association that rules out the confounding factors found in the current study. Matching individual scores on culture, climate, and the implementation of TIC practices, policies and procedures will enable a more sophisticated model of the unique intervention effects to be explored. Future research will also examine a broader array of outcomes. As noted, the implementation of TIC is expected to increase employee retention, enhance organizational commitment, and may lead to increases in 
organizational performance: these hypotheses will be tested in future research. In regards to client outcomes, due to the emphasis on collaborative and participative practices, the implementation of TIC is expected to positively influence the therapeutic alliance. Therapeutic alliance refers to the strength of the relationship between clients and directcare staff (Bordin, 1979). Enhancement of the therapeutic alliance has been positively associated with retention in treatment and improvement in mental health outcomes (Martin, Garske, \& Davis, 2000). These relationships will be tested in future research.

\section{References}

Azeem, M. W., Aujla, A., Rammerth, M., Binsfeld, G., \& Jones, R. B. (2011).

Effectiveness of six core strategies based on trauma informed care in reducing seclusions and restraints at a child and adolescent psychiatric hospital. Journal of Child and Adolescent Psychiatric Nursing, 24(1), 11-15. doi: https://doi.org/10.1111/j.1744-6171.2010.00262.x

Babin, B. J., \& Boles, J. S. (1996). The effects of perceived co-worker involvement and supervisor support on service provider role stress, performance and job satisfaction. Journal of Retailing, 72(1), 57-75. doi: https://doi.org/10.1016/S00224359(96)90005-6

Bloom, S. L. (2013). Creating sanctuary: Toward the evolution of sane societies. New York, NY: Routledge.

Bordin, E. S. (1979). The generalizability of the psychoanalytic concept of the working alliance. Psychotherapy: Theory, Research \& Practice, 16(3), 252-260. doi: https://doi.org/10.1037/h0085885

Brown, S. P., \& Peterson, R. A. (1993). Antecedents and consequences of salesperson job satisfaction. Meta-analysis and assessment of causal effects. Journal of Marketing Research, 30(1), 63-77. doi: https://doi.org/10.2307/3172514

Cameron, K. S., \& Quinn, R. E. (2005). Diagnosing and changing organizational culture: Based on the competing values framework. San Francisco, CA: Jossey-Bass.

Centers for Disease Control and Prevention. (n.d.). About the CDC-Kaiser ACE study. Retrieved from https://www.cdc.gov/violenceprevention/acestudy/about.html

Esaki, N., Benamati, J., Yanosy, S., Middleton, J., Hopson, L., Hummer, V., \& Bloom, S. (2013). The sanctuary model: Theoretical framework. Families in Society: The Journal of Contemporary Social Services, 94(2), 87-95. doi: https://doi.org/10.1606/1044-3894.4287

Fallot, R. D., \& Harris, M. (2008). Trauma-informed approaches to systems of care. Trauma Psychology Newsletter, 3(1), 6.

Felitti, V. J., Anda, R. F., Nordenberg, D., Williamson, D. F., Spitz, A. M., Edwards, V., ... Marks, J. S. (1998). Relationship of childhood abuse and household dysfunction to many of the leading causes of death in adults: The Adverse Childhood Experiences 
(ACE) Study. American journal of preventive medicine, 14(4), 245-258. doi: https://doi.org/10.1016/S0749-3797(98)00017-8

Griffin, M. A., Patterson, M. G., \& West, M. A. (2001). Job satisfaction and teamwork: The role of supervisor support. Journal of Organizational Behavior, 22(5), 537-550. doi: https://doi.org/10.1002/job.101

Guarino, K., Soares, P., Konnath, K., Clervil, R., \& Bassuk, E. (2009). Trauma-informed organizational toolkit. Rockville, MD: Center for Mental Health Services, Substance Abuse and Mental Health Services Administration, and the Daniels Fund, the National Child Traumatic Stress Network, and the W.K. Kellogg Foundation. Retrieved from www.homeless.samhsa.gov

Hales, T., Kusmaul, N., \& Nochajski, T. (2017). Exploring the dimensionality of traumainformed care: Implications for theory and practice. Human Service Organizations: Management, Leadership \& Governance, 41(3), 317-325. doi: https://doi.org/10.1080/23303131.2016.1268988

Harris, M. E., \& Fallot, R. D. (2001). Using trauma theory to design service systems. San Francisco, CA: Jossey-Bass.

Hodgdon, H. B., Kinniburgh, K., Gabowitz, D., Blaustein, M. E., \& Spinazzola, J. (2013). Development and implementation of trauma-informed programming in youth residential treatment centers using the ARC framework. Journal of Family Violence, 28(7), 679-692. doi: https://doi.org/10.1007/s10896-013-9531-Z

Kilpatrick, D. G., Resnick, H. S., Milanak, M. E., Miller, M. W., Keyes, K. M., \& Friedman, M. J. (2013). National estimates of exposure to traumatic events and PTSD prevalence using DSM-IV and DSM-5 criteria. Journal of Traumatic Stress, 26(5), 537-547. doi: https://doi.org/10.1002/jts.21848

Korunka, C., Scharitzer, D., Carayon, P., \& Sainfort, F. (2003). Employee strain and job satisfaction related to implementation of quality in a public service organization: A longitudinal study. Work \& Stress, 17(1), 52-72. doi: https://doi.org/10.1080/0267837031000109526

Koys, D. J. (2001). The effects of employee satisfaction, organizational citizenship behavior, and turnover on organizational effectiveness: A unit-level, longitudinal study. Personnel Psychology, 54(1), 101-114. doi: https://doi.org/10.1111/j.1744$\underline{6570.2001 . t b 00087 . x}$

Kramer, T. L., Sigel, B. A., Conners-Burrow, N. A., Savary, P. E., \& Tempel, A. (2013). A statewide introduction of trauma-informed care in a child welfare system. Children and Youth Services Review, 35(1), 19-24. doi: https://doi.org/10.1016/j.childyouth.2012.10.014

Kuo, J. R., Goldin, P. R., Werner, K., Heimberg, R. G., \& Gross, J. J. (2011). Childhood trauma and current psychological functioning in adults with social anxiety disorder. Journal of Anxiety Disorders, 25(4), 467-473. doi: https://doi.org/10.1016/j.janxdis.2010.11.011 
Kusmaul, N., Wilson, B., \& Nochajski, T. (2015). The Infusion of trauma-informed care in organizations: Experience of agency staff. Human Service Organizations: Management, Leadership \& Governance, 39(1), 25-37. doi: https://doi.org/10.1080/23303131.2014.968749

LeBel, J., \& Goldstein, R. (2005). Special section on seclusion and restraint: The economic cost of using restraint and the value added by restraint reduction or elimination. Psychiatric Services, 56(9), 1109-1114. doi:

https://doi.org/10.1176/appi.ps.56.9.1109

LeBel, J., Stromberg, N., Duckworth, K., Kerzner, J., Goldstein, R., Weeks, M., ... Sudders, M. (2004). Child and adolescent inpatient restraint reduction: A state initiative to promote strength-based care. Journal of the American Academy of Child \& Adolescent Psychiatry, 43(1), 37-45. doi: https://doi.org/10.1097/00004583200401000-00013

Madsen, L. H., Blitz, L. V., McCorkle, D., \& Panzer, P. G. (2003). Sanctuary in a domestic violence shelter: A team approach to healing. Psychiatric Quarterly, 74(2), 155-171. doi: https://doi.org/10.1023/A:1021307811184

Martin, D. J., Garske, J. P., \& Davis, M. K. (2000). Relation of the therapeutic alliance with outcome and other variables: A meta-analytic review. Journal of Consulting and Clinical Psychology, 68(3), 438-450. doi: https://doi.org/10.1037/0022$\underline{\text { 006X.68.3.438 }}$

Meyer, J. P, Stanley, D. J., Herscovitch, L., \& Topolnytsky, L. (2002). Affective, continuance, and normative commitment to the organization: A meta-analysis of antecedents, correlates, and consequences. Journal of Vocational Behavior, 61(1), 20-52. doi: https://doi.org/10.1006/jvbe.2001.1842

Morrissey, J. P., Jackson, E. W., Ellis, A. R., Amaro, H., Brown, V. B., \& Najavits, L. M. (2005). Twelve-month outcomes of trauma-informed interventions for women with co-occurring disorders. Psychiatric Services, 56(10), 1213-1222. doi: https://doi.org/10.1176/appi.ps.56.10.1213

Muskett, C. (2014). Trauma-informed care in inpatient mental health settings: A review of the literature. International Journal of Mental Health Nursing, 23(1), 51-59. doi: https://doi.org/10.1111/inm.12012

Najavits, L. M. (2002). Seeking safety: A treatment manual for PTSD and substance abuse. NY: Guilford Press.

Najavits, L. M., Weiss, R. D., Shaw, S. R., \& Muenz, L. R. (1998). "Seeking safety": Outcome of a new cognitive-behavioral psychotherapy for women with posttraumatic stress disorder and substance dependence. Journal of Traumatic Stress, 11(3), 437456. doi: https://doi.org/10.1023/A:1024496427434

Park, S., Hong, J. P., Bae, J. N., Cho, S. J., Lee, D. W., Lee, J. Y., ... Seong, S. (2014). Impact of childhood exposure to psychological trauma on the risk of psychiatric disorders and somatic discomfort: Single vs. multiple types of psychological trauma. 
Psychiatry Research, 219(3), 443-449. doi:

https://doi.org/10.1016/j.psychres.2014.06.009

Resick, P. A., Nishith, P., Weaver, T. L., Astin, M. C., \& Feuer, C. A. (2002). A comparison of cognitive-processing therapy with prolonged exposure and a waiting condition for the treatment of chronic posttraumatic stress disorder in female rape victims. Journal of Consulting and Clinical Psychology, 70(4), 867-879. doi: https://doi.org/10.1037/0022-006X.70.4.867

Seidler, G. H., \& Wagner, F. E. (2006). Comparing the efficacy of EMDR and traumafocused cognitive-behavioral therapy in the treatment of PTSD: A meta-analytic study. Psychological Medicine, 36(11), 1515-1522. doi: https://doi.org/10.1017/S0033291706007963

Somers, M. J. (1995). Organizational commitment, turnover and absenteeism: An examination of the direct and interaction effects. Journal of Organizational Behavior, 16(1), 49-58. doi: https://doi.org/10.1002/job.4030160107

Strand, V. C., \& Dore, M. M. (2009). Job satisfaction in a stable state child welfare workforce: Implications for staff retention. Children and Youth Services Review, 31(3), 391-397. doi: https://doi.org/10.1016/j.childyouth.2008.09.002

Strand, V. C., Spath, R., \& Bosco-Ruggiero, S. (2010). So you have a stable child welfare workforce-What's next? Children and Youth Services Review, 32(3), 338-345. doi: https://doi.org/10.1016/j.childyouth.2009.10.002

Substance Abuse and Mental Health Services Administration. (2014). SAMHSA's concept of trauma and guidance for a trauma-informed approach. HHS Publication No. (SMA) 14-4884. Rockville, MD: Substance Abuse and Mental Health Services Administration.

WorkplaceDynamics. (n.d.). Business Insight Report. Retrieved from: http://www.workplacedynamics.com/pdf/SampleCo_2016_Business_Insight_Report.pdf

Author note:

Address correspondence to: Travis Hales, School of Social Work, University at Buffalo, 219 Parker Hall, University at Buffalo, South Campus, Buffalo, NY 14214-8004, 716 829 5857, twhales@buffalo.edu 